\section{Arguing (About) the Value of Cancer Care}

\author{
Ivo Abraham, PhD, RN; Ali McBride, MS, PharmD; and \\ Karen MacDonald, PhD, RN
}

The debate about the escalating cost of caring for patients with cancer may soon derail into an argument (as in, dispute) that will not help those providing care, those developing treatments, and those paying for care. In the process, the voice of patients-as co-decision-maker, recipient, and copayer of this care-may grow even fainter. Pointing out what others (presumably) do wrong, (presumably) fail to do, or (presumably) should do differently to stem the rising costs of cancer treatment is easy. Building a collective argument (as in, case) that balances the interests and concerns of all stakeholders - and does so in a clinically responsible, economically accountable, and patient-centered manner-is much harder.

Some hard questions must be answered by all stakeholders. These are our thoughts on them.

\section{Does the United States Overspend on Drugs?}

Proportionately, the United States does not overspend on drugs, as data from 32 Organization for Economic Cooperation and Development (OECD) countries suggest. ${ }^{1}$ In 2014, 12.3\% of total healthcare expenditures in the United States was for pharmaceuticals and other medical "nondurables," ranking 24. This $12.3 \%$ was about the same as the United Kingdom (12.1\%); less than Germany (14.5\%), France (15.0\%), Italy (17.0\%), Canada (17.2\%), Spain (17.9\%), and Japan (18.9\% [2013 data]); but more than Denmark (6.7\%), Norway (7.5\%), and Sweden (9.8\%).

These countries may have more restricted formularies, especially in cancer drugs and biological agents, and lower drug prices in general. This deflates medication costs relative to the United States. Higher percentages may indeed hide comparably higher pharmaceutical expenditures. Other drivers of healthcare costs prevail more strongly in the United States, such as labor, facilities, service mix, compliance, and consumer expectations, among others.

\section{Should We Focus Solely on Cost?}

This is perhaps a rhetorical question, but if it were only a matter of cost containment, the solution to the evolving crisis is straightforward (price controls), forced down by one stakeholder (the majority payer), enforced by that same stakeholder, and driven by what this stakeholder takes in, is willing to pay out, and decides what to do with the difference. Admittedly, this is an unlikely scenario. Cost containment measures are necessary, however. If all stakeholders are engaged, quality of care and outcomes need not be impaired and responsible care could be rewarded. Take the Oncology Care Model currently being piloted by the Centers for Medicare \& Medicaid Servies (CMS). In this model, provider organizations are paid for chemotherapy services on the basis of performance and financial accountability, including enhanced services such as care coordination and navigation, adherence to evidence-based treatment guidelines, and assuring access to care. ${ }^{2}$ Although not without critics, ${ }^{3}$ the Oncology Care Model is an example of the shift from (unilateral) cost reduction to balancing cost containment with value.

\section{Do We Have a Workable Definition of Value in Cancer Care?}

We do have a definition of value in healthcare, but whether it is workable in cancer remains to be seen. Porter ${ }^{4}$ defines value as the health outcomes achieved per dollar spent, distinguishing between 3 hierarchical tiers of outcomes, each with 2 levels. Tier

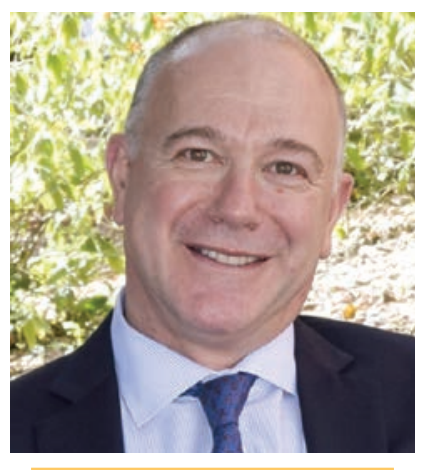

Ivo Abraham, PhD, RN

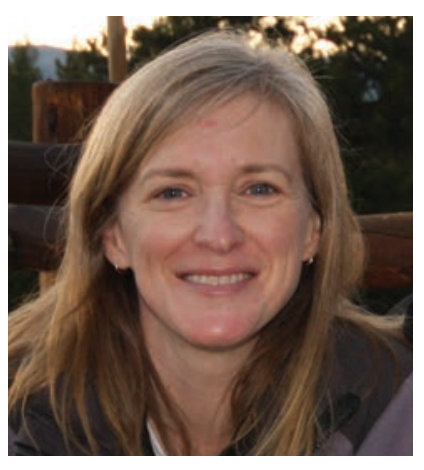

Karen MacDonald, PhD, RN Ivo Abraham, PhD, RN, is Professor of Pharmacy and Medicine at the University of Arizona, where he is also the Director of the Center for Health Outcomes and PharmacoEconomic Research and a scientific member of the University of Arizona Cancer Center, and also serves as Chief Scientist of Matrix45 (all in Tucson, AZ). Karen MacDonald, $\mathrm{PhD}, \mathrm{RN}$, is the Managing Director of Matrix45, a scientific consulting company specializing in clinical real-world research. As outcomes and effectiveness researchers, they focus on how in real-world clinical practice variation in treatment patterns is associated with variations in clinical,

patient-centered, and economic outcomes; the patient, clinician, and setting factors that influence these variations in treatment and in outcomes; and the variance in outcomes that can be attributed to patient, clinician, and setting. As clinician-methodologists, they work across several therapeutic areas, but for the past $15+$ years have focused extensively on real-world cancer care, first in supportive cancer care and later evolving also into curative cancer care in the real-world setting. With the approval of the first biosimilars in Europe almost a decade ago, they designed and supported the first large-scale studies on their safety, effectiveness, and economics. They also have an interest in quantifying the extent to which the cancer care of individual patients is congruent with evidence-based guidelines and how this translates into improved outcomes.

The ideas and viewpoints expressed in this commentary are those of the autho and do not necessarily represent any
policy, position, or program of NCCN. 


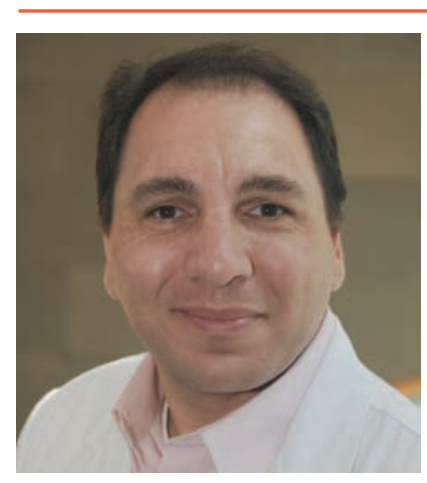

Ali McBride, MS, PharmD

Ali McBride, MS, PharmD, is the Clinical Pharmacy Coordinator for Oncology/Hematology at Banner University Medical Center and the University of Arizona Cancer Center where he also serves as a scientific member; and Assistant Professor of Pharmacy at the University of Arizona (Tucson, AZ). He has worked for more than 10 years in clinical practice addressing cancer care for patients and assessing supportive care in clinics. He currently serves as the Secretary of Association of Community Cancer Centers and serves on the National Quality Forum on Cancer.
1 refers to the health status achieved or retained, distinguishing between the levels of survival and the degree of health or recovery. Tier 2 concerns the process of recovery, and differentiates between the time to recovery and return to normal activities, and the disutility of care or treatment process due to adverse outcomes. Tier 3 involves the sustainability of health; the first level refers to the absence of recurrences of the original disease or its long-term implications, and the second level to the long-term consequences of therapy and care-induced illnesses in particular.

Conceptually, the Porter model has all the elements that intuitively are important to cancer: outcomes ranging from long-term disease-free survival and full functional status, to variations in recovery or decline, achieved over gradients of time and adjusted downward for adverse outcomes due to clinical practice or treatment, down to longer-term cancer recurrence, second cancers, or treatment-related consequences. Yet, for the Porter model to be helpful in cancer, it will need to risk-adjust dynamically over time for type of cancer, stage of disease, treatment options, prognosis, efficacy promises from trials, effectiveness outcomes from real-world practice, short- and longterm treatment-related consequences and complications, and patient acceptance of these. It will also need to accommodate changing patient preferences and clinician decision-making when guidelines are suggestive rather than normative or when realworld patients differ from trial-world patients.

\section{Do We Have the Tools to Quantify Value in Cancer Care?}

We do not currently have the tools to quantify value, and that is why a shorterterm focus might be indicated (eg, on episodes of care and a reasonable postepisode observation period). In this, we need to use parameters that can be harmonized in definition and metrics, where outcomes are assessed against various time horizons and where costs can be determined accurately. This is where the pragmatic efforts by ASCO, ${ }^{5,6} \mathrm{ESMO},{ }^{7}$ and NCCN may prove helpful to all stakeholders.

In an excellent review on the work of these organizations (and the Institute for Clinical and Economic Review and Memorial Sloan Kettering Cancer Center Drug Abacus), Schnipper and Bastian ${ }^{8}$ point out that the ASCO and ESMO frameworks attempt to quantify gradients of clinical benefit of drug regimens, toxicity, and quality of life-elements also central to the NCCN model. The ASCO framework reconciles the net health benefit (NHB) of a treatment with its cost to payer and patient, where NHB is defined as clinical benefit adjusted downward for toxicity and upward for extended survival benefit, palliation, quality of life, and treatment-free interval. The ESMO approach emphasizes "living longer," either overall or disease-free, and, though to a lesser extent, "living better" by considering improvements in quality of life and reductions in toxicity. It does not address cost and value. Building on its extensive library of guidelines and covering all treatments for cancer, the NCCN model aligns effectiveness and safety parameters with the quality and consistency of the evidence and the affordability of treatment options.

\section{Should We Integrate Patient Experience, and How?}

We believe we should integrate patient experience, but all stakeholders may not agree on how. The ASCO, ESMO, and NCCN approaches consider quality of life but do not advocate use of the quality-adjusted life year (QALY), something the International Society for Pharmacoeconomics and Outcomes Research challenged regarding the ASCO framework. ${ }^{9}$ A widely used metric, QALY adjusts the hypothetical years lived in (hypothetical) perfect health for the burden of disease and treatment by applying utility values. When comparing treatment options, it quantifies the incremental QALYs gained by a superior over a comparator treatment. The QALY metric may be helpful in economic evaluations from the societal perspective, whether as general concept ("for 
the good of society") or in single-payer systems in which cost-effectiveness thresholds are applied to determine coverage and reimbursement (eg, the United Kingdom). QALYs are less relevant in multipayer health systems. The application of thresholds can be questioned in terms of their evidence base, the relative lack of adjustment for inflation, and the tendency for "threshold creep."

Although speculation on our part, the absence of QALYs in the ASCO, ESMO, and NCCN models may also be due to the relatively abstract, if not artificial nature, of the concept. Certainly, using the QALY concept in the clinic to answer the questions patients are most likely to ponder is difficult. These questions include: How much longer will I live? How hard will it be to achieve that? How well will I live? What will I have to pay? Is this worth it?

\section{Should We Make Exceptions?}

We believe that we should not make exceptions in determining value but instead should adjust for cancer case mix, scope of services, and additional responsibilities of provider organizations. We should also adjust for patient factors that impair treatment outcomes, such as genetics and nonadherence. Just recently, JNCCN published the report of an NCCN work group examining the value, access, and cost of cancer care delivery at academic cancer centers. ${ }^{10}$ Although some may perceive the report as a somewhat defensive justification of the higher costs of care at academic cancer centers, the work group substantiated its position with evidence of superior survival benefits at academic cancer centers, while also considering such factors as patient experience, access to care, and health disparities. Noteworthy also is the report's comparison of the differences in scope and responsibilities of community and academic cancer care providers.

\section{What Next?}

As we move forward, foremost stakeholders will need (or be forced) to accept that the economics of cancer care today are the consequence of our culture and society. Stakeholders will need to come together on what is worth treating and what is not, and what is worth paying for and what is not. Realistically, the cost of cancer will continue to rise. Inequities and disparities seen from current cost containment and rationing methods are likely to grow worse unless clinically relevant and economically sustainable care models, accessible to all, are implemented. This accessibility is economic, and therefore should be economically fair. Patient participation in paying for care is essential but should be graded by ability to pay, lest patient copay become the equivalent of a regressive tax on seeking treatment for cancer.

\section{References}

1. Organisation for Economic Co-operation and Development. OECD Health Statistics 2016. Frequently requested data. Available at: http://www.oecd.org/els/health-systems/health-data.htm. Accessed September 30, 2016.

2. Kline RM, Bazell C, Smith E, et al. Centers for Medicare and Medicaid Services: using an episode-based payment model to improve oncology care. J Oncol Pract 2015;11:114-116.

3. Polite BN, Miller HD. Medicare innovation center oncology care model: a toe in the water when a plunge is needed. J Oncol Pract 2015;11:117-119.

4. Porter ME. What is value in healthcare? N Engl J Med 2010;363:2477-2481.

5. Schnipper LE, Davidson NE, Wollins DS, et al. American Society of Clinical Oncology statement: a conceptual framework to assess the value of cancer treatment options. J Clin Oncol 2015;33:2563-2577.

6. Schnipper LE, Davidson NE, Wollins DS, et al. Updating the American Society of Clinical Oncology Value Framework: revisions and reflections in response to comments received. J Clin Oncol 2016;34:2925-2934.

7. Cherny NI, Sullivan R, Dafni U, et al. A standardised, generic, validated approach to stratify the magnitude of clinical benefit that can be anticipated from anti-cancer therapies: the European Society for Medical Oncology Magnitude of Clinical Benefit Scale (ESMO-MCBS) [published online ahead of print September 7, 2016]. Ann Oncol 2016, doi: 10.1093/annonc/mdv249.

8. Schnipper LE, Bastian A. New frameworks to assess value of cancer care: strengths and limitations. Oncologist 2016;21:654-658.

9. Malone DC, Berg NS, Claxton LP, et al. International Society for Pharmacoeconomics and Outcomes Research comments on the American Society of Clinical Oncology Value Framework. J Clin Oncol 2016;34:2936-2937.

10. Nardi EA, Wolfson JA, Rosen ST, et al. Value, access, and cost of cancer care delivery at academic cancer centers. J Nat Compr Canc Netw 2016;14;837-847. 